\title{
simulation numérique du champ de contraintes au sein de matières granulaires ensilées - comparaison avec les mesures in situ
}

\author{
numerical simulation of the stress field inside ensiled materials - \\ comparative study with in situ experimental results
}

\author{
J.-C. REMESCH* ${ }^{*}$, G. MAURICE* ", A. KHELIL*, J.-C. ROTH* \\ Laboratoire d'Energétique et de Mécanique Théorique et Appliquée (LEMTA)
}

Rev. Franç. Géotech. n 52, pp. 51-65 (juillet 1990)

\section{Résumé}

L'article présente une simulation numérique des contraintes dans les silos métalliques à partir du programme d'éléments finis SILO (Université de Karlsruhe) utilisant les lois de comportement des milieux granulaires de P.V. LADE et de D. KOLYMBAS. Les résultats numériques sont comparés aux résultats expérimentaux obtenus dans les silos réels de la base de Chartres. L'influence des paramètres géométriques des silos et des caractéristiques mécaniques de la matière ensilée sur les contraintes pariétales complète cette étude.

\section{Abstract}

This paper give a numerical simulation of the stresses in steel silos using behaviour laws of P.V. LADE and D. KOLYMBAS. The numerical results computed by the software SILO (University of Karlsruhe) are compared with the experimental results obtained in the "silos base of Chartres". We examine the effect of various characteristics parameters of the silo and the mechanical properties of the ensiled materials on the wall stresses. 


\section{INTRODUCTION}

L'analyse du comportement des milieux granulaires utilise, la plupart du temps, des lois de comportement dérivées de l'étude de la mécanique des sols. Parmi ces lois signalons les modèles utilisant la plasticité et le concept d'état critique, les lois élastoplastiques (CAMBOU, LADE...), les lois hypoélastiques (DAVID TEAM, COLLINS-BACHUS, KOLYMBAS ...) et les lois incrémentales (DARVE, MIURA-FINN...). Ces différentes lois sont analysées et comparées par CAMBOU [2]. Les lois de comportement de P.V. LADE [12] et de D. KOLYMBAS [10] sont les seules actuellement intégrées dans le programme "SILO * [14] pour la simulation des contraintes dans les matières ensilées.

Notre étude a pour objectif de comparer les résultats expérimentaux obtenus dans les silos du site de Chartres [1] et les prévisions numériques obtenues par éléments finis à partir du programme SILO [14] utilisant l'une ou l'autre de ces lois. Les conditions aux limites au niveau du matériau et des parois de la cellule sont adaptées conformément aux indications données par U. HAUSSLER [7] et W. GLADEN [6].

Nous présentons d'abord brièvement les caractéristiques de chacune des lois de comportement et la manière de déterminer expérimentalement leurs paramètres. Après avoir précisé la nature des essais expérimentaux effectués nous nous intéressons aux résultats provenant des approches expérimentales et numériques afin de mieux situer ces deux lois de comportement dans le contexte de leur utilisation pratique.

\section{LA LOI DE LADE}

Le modèle développé initialement par LADE pour les sols sans cohésion, peut être étendu au domaine plus vaste des milieux pulvérulents (sables, poudres, milieux granulaires)

Ce modèle fait appel aux concepts d'élasticité et de plasticité du matériau. Les aspects visqueux ne sont, par contre, pas pris en compte.

Le modèle rhéologique de LADE contient 14 paramètres caractéristiques du matériau qui peuvent être entièrement déterminés à partir de deux types d'essais en laboratoire : des essais de compression isotrope et des essais conventionnels drainés de compression triaxiale.

L'analyse du comportement des sols sans cohésion a conduit à diviser l'incrément de déformation totale $\mathrm{d} \epsilon_{\mathrm{ij}}$ en une composante élastique $\left(\mathrm{d} \epsilon_{\mathrm{ji}}\right)_{\mathrm{e}}$, une composante plastique de consolidation $\left(d \epsilon_{f}\right)_{c}$ et une composante plastique de distorsion $\left(\mathrm{d} \epsilon_{\mathrm{ij}}\right)_{\mathrm{p}}$ soit :

$$
\mathrm{d} \epsilon_{\mathrm{ij}}=\left(\mathrm{d} \epsilon_{\mathrm{ij}}\right)_{e}+\left(\mathrm{d} \epsilon_{\mathrm{i}}\right)_{\mathrm{c}}+\left(\mathrm{d} \epsilon_{\mathrm{ij}}\right)_{\mathrm{p}}
$$

Ces trois composantes sont déterminées séparément : - la composante élastique $\left(\mathrm{d}_{\epsilon_{\mathrm{ij}}}\right)_{\mathrm{e}}$ à partir de la loi de HOOKE ;
- la composante plastique de consolidation $\left(\mathrm{d} \epsilon_{i i}\right)$ cà l'aide d'une théorie contrainte-déformation incluant une surface de charge sphérique centrée à l'origine de l'espace des contraintres principales et un potentiel plastique associé ;

- la composante plastique de dilatation $\left(\mathrm{d} \epsilon_{\mathrm{ij}}\right)_{\mathrm{p}}$ à l'aide d'une relation contrainte-déformation incluant une surface de charge conique ayant pour sommet l'origine de l'espace des contraintes principales et un potentiel plastique non associé.

La figure 1 montre schématiquement les différentes parties de la déformation totale considérées par LADE au cours d'un essai triaxial.

\subsection{Déformations élastiques}

Elles sont recouvrables après déchargment et déterminées par la loi de HOOKE

$$
[\mathrm{d} \epsilon]_{e}=\left[\mathrm{D}_{e}\right]^{-1}[\sigma]
$$

$\left[D_{e}\right]$ représente la matrice de rigidité élastique faisant intervenir le module d'YOUNG E et le coefficient de POISSON $y$ du matériau. Le module d'YOUNG dépend de la pression de confinement $\sigma_{3}$ et de la pression atmosphérique $\mathrm{P}_{\mathrm{a}}$ selon une loi de type puissance :

$$
E=K P_{a}\left(\frac{\sigma_{3}}{P_{a}}\right)^{n}
$$

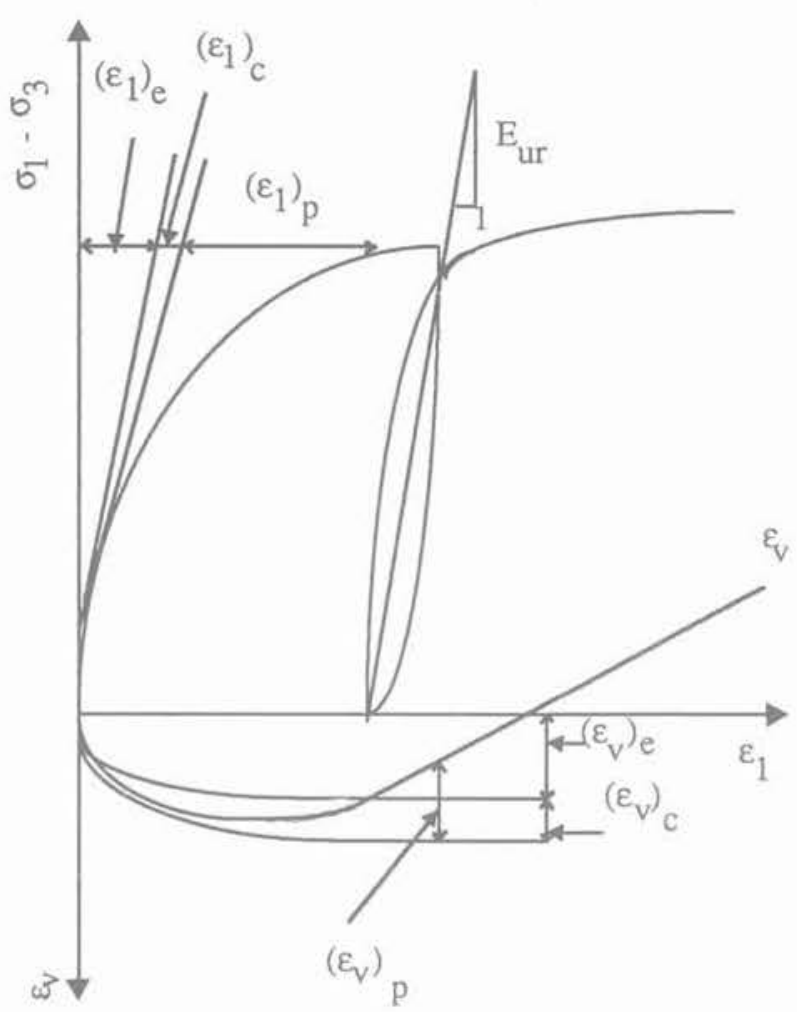

Fig. 1. - Illustration schématique des déformations : élastique, plastique de consolidation et plastique de distorsion dans un essai triaxial.

Fig. 1. - Schematic illustration of elastic, plastic collapse and plastic expansive strain components in triaxial compression test. 
$\mathrm{K}$ et $\mathrm{n}$ sont des nombres sans dimension que l'on détermine expérimentalement à partir d'essais triaxiaux de compression réalisés pour diverses valeurs de $\sigma_{3}$. La valeur du coefficient de Poisson $\nu$ est en général voisine de 0,2 pour le type de matériaux granulaires qui nous intéresse.

\subsection{Déformations plastiques de consolidation}

On a constaté expérimentalement qu'une partie des déformations obtenues au cours d'une compression isotrope n'était pas recouvrable, donc plastique. Il est raisonnable de penser que ces déformations plastiques résultent d'une augmentation de la contrainte moyenne. Il est alors logique d'associer les contraintes de cisaillement aux déformations plastiques de distorsion. Cependant en pratique, les déformations plastiques de consolidation et les déformations plastiques de distorsion se produisent simultanément.

Il est donc nécessaire de déterminer la valeur des déformations de consolidation à partir d'un essai de compression isotrope, car ceci est le seul cas de chargement qui n'introduit pas de déformation plastique de distorsion.

\subsubsection{Surface de charge associée aux déformations plastiques de consolidation}

La surface de charge retenue pour le modele a la forme d'une sphère centrée sur l'origine de l'espace des contraintes principales $\sigma_{1}, \sigma_{2}, \sigma_{3}$. Son équation, $\mathrm{Fc}=0$, est définie à partir du premier invariant $\mathrm{I}_{1}=\sigma_{1}+\sigma_{2}+\sigma_{3}$ et du second invariant :

$I_{2}=-\left(\sigma_{1} \sigma_{2}+\sigma_{3} \sigma_{2}+\sigma_{3} \sigma_{1}\right)$ du tenseur des contraintes. Elle s'exprime par:

$$
\mathrm{Fc}=\mathrm{I}_{1}{ }^{2}+2 \mathrm{I}_{2}-\mathrm{f}_{\mathrm{c}}=0
$$

où $\mathrm{f}_{\mathrm{c}}$ représente le degré de consolidation défini par P.V. LADE [12].

\subsubsection{Potentiel plastique et loi d'écoulement}

La compression isotrope d'un matériau isotrope provoque des déformations égales suivant les trois directions principales ce qui implique un potentiel plastique associé égal à la fonction de charge. La règle d'écoulement s'écrit donc :

$$
\left(d \epsilon_{i \mathrm{ij}}\right)_{c}=\Delta \lambda_{c}\left(\frac{\partial f_{c}}{\partial \sigma_{\mathrm{ij}}}\right)
$$

où $\Delta \lambda_{c}$ est le multiplicateur de plasticité associé aux déformations plastiques de consolidation.

\subsubsection{Ecrouissage positif}

Pour déterminer les déformations plastiques de consolidation à partir de l'état de contraintes, il faut déterminer $\Delta \lambda_{c}$. Cela peut se faire à l'aide de la loi d'écrouissage de la surface de charge relative aux déformations plastiques de consolidation proposée par LADE et qui s'écrit :

$$
W_{c}=F\left(f_{c}\right)=C P_{a}\left(\frac{f_{c}}{P_{a}^{2}}\right)^{p}
$$

où $\mathrm{C}$ est le module de consolidation et $\mathrm{p}$ l'exposant de consolidation. $W_{c}$ est le travail plastique nécessaire pour produire les déformations plastiques de consolidation.

$$
W_{c}=\int\left(\sigma_{i j}\right)^{t}\left(\mathrm{~d} \epsilon_{\mathrm{ij}}\right)_{c}
$$

La courbe expérimentale liant le travail plastique $W_{c}$ et le degré de consolidation $\mathrm{f}_{\mathrm{c}}$ est déterminée à l'aide d'essais de compressions isotropes. $\Delta \lambda_{c}$ est obtenu par les expressions ci-dessous :

$$
\begin{aligned}
& \Delta \lambda_{c}=\left(\frac{d W_{c}}{2 f_{c}}\right) \\
& \text { et } d W_{c}=p C P_{a}\left(\frac{f_{c}}{P_{a}^{2}}\right)^{p-1} d\left(\frac{f_{c}}{P_{a}{ }^{2}}\right)
\end{aligned}
$$

\subsection{Déformations plastiques de distorsion}

L'expérience montre que les déformations plastiques de contraction se produisent sous faible niveau de déviateur de contraintes. Elles ne deviennent de distorsion que sous haut niveau de déviateur de contraintes. Le modèle considère cependant ces déformations plastiques comme étant toujours de distorsion.

\subsubsection{Surface de rupture et surface de charge associées aux déformations plastiques de distorsion}

La surface de rupture est courbe pour la plupart des sols, plus précisément l'angle de frottement interne décroit avec laugmentation de la contrainte moyenne. La fonction de charge proposé par LADE s'écrit :

$$
F_{p}=\left(\frac{I_{1}^{3}}{I_{3} \cdot}-27\right)\left(\frac{I_{1}}{P_{a}}\right)^{m}-f_{p}=0
$$

$\mathrm{f}_{\mathrm{p}}$ est une fonction représentant le degré d'écrouissage du matériau. La surface de rupture est la position ultime de la surface de charge. Soit $\eta_{1}$ la valeur limite de $\mathrm{f}_{\mathrm{p}}$ à l'état de rupture [12].

$$
\eta_{1}=\left(\frac{\mathrm{I}_{1}^{3}}{\mathrm{I}_{3}}-27\right)\left(\frac{\mathrm{I}_{1}}{\mathrm{P}_{\mathrm{a}}}\right)^{\mathrm{m}}
$$

\subsubsection{Potentiel plastique et loi d'écoulement}

Le potentiel plastique relatif aux déformations de dilatation est non associé, et défini par la relation :

$$
\mathrm{g}_{\mathrm{p}}=\mathrm{I}_{1}{ }^{3}-\mathrm{I}_{3}\left[27+\eta_{2}\left(\frac{\mathrm{P}_{\mathrm{a}}}{\mathrm{I}_{1}}\right)^{\mathrm{m}}\right]
$$

où $\eta_{2}$ et $\mathrm{m}$ sont des paramètres à déterminer. La loi d'écoulement est donnée par l'équation :

$$
\left(d \epsilon_{i j}\right)_{p}=\Delta \lambda_{p} \frac{\partial g_{p}}{\partial \sigma_{i j}}
$$

où $\Delta \lambda_{\mathrm{p}}$ est le multiplicateur de plasticité associé aux déformations plastiques de distorsion. 
La valeur de $\eta_{2}$ peut théoriquement être calculée à partir d'un essai de compression triaxiale par les relations suivantes.

$\eta_{2}=\left[\frac{3\left(1+\nu_{\mathrm{p}}\right) \mathrm{I}_{1}{ }^{2}-27 \sigma_{3}\left(\sigma_{1}+\nu_{\mathrm{p}} \sigma_{3}\right)}{\left(\frac{\mathrm{P}_{\mathrm{a}}}{\mathrm{I}_{1}}\right)^{\mathrm{m}}\left[\sigma_{3}\left(\sigma_{1}+\nu_{\mathrm{p}} \sigma_{3}\right)-\frac{\mathrm{m}\left(1+\nu_{\mathrm{p}}\right) \mathrm{I}_{1}{ }^{2}}{\mathrm{f}_{\mathrm{p}}\left(\frac{\mathrm{P}_{\mathrm{a}}}{\mathrm{I}_{1}}\right)^{\mathrm{m}}+27}\right.}\right]$

où

$$
\nu_{\mathrm{p}}=-\frac{\left(\mathrm{d} \epsilon_{3}\right)_{\mathrm{p}}}{\left(\mathrm{d} \epsilon_{1}\right)_{\mathrm{p}}}
$$

qu'on peut écrire sous la forme:

$$
\eta_{2}=S f_{p}+R \sqrt{\frac{\sigma_{3}}{P_{a}}}+T
$$

$\mathrm{R}$ et $\mathrm{T}$ sont des constantes à déterminer.

Dans la pratique les dispersions dues aux erreurs expérimentales conduit à ne retenir comme valeur correcte qu'une moyenne de ces relevés expérimentaux.

\subsubsection{Ecrouissage négatif}

Dans un écrouissage négatif, le matériau présente un ramollissement au-delà d'un certain pic. Le modèle de LADE utilise des surfaces de charge associées à un degré d'écrouissage $f_{p}$ indépendant de l'histoire des sollicitations. L'hypothèse d'écrouissage isotrope, conduit à une relation unique entre le travail plastique $W_{p}$ :

$$
\mathrm{W}_{\mathrm{P}}=\int\left(\sigma_{\mathrm{ij}}\right)^{\mathrm{t}}\left(\mathrm{d} \epsilon_{\mathrm{ij}}\right)_{\mathrm{P}}
$$

et le degré d'écrouissage $\mathrm{f}_{\mathrm{p}}$.

LADE considère une relation mathématique de la forme $W_{p}=F_{p}\left(f_{p}\right)$ où $F_{p}$ est une fonction positive initialement croissante jusqu'à une valeur maximale $W_{\text {pmax }}$, puis décroissante au-delà de ce pic. La relation mathématique inverse de $F_{p}$ est donnée par l'expression simple suivante:

$$
f_{p}=a e^{-b W_{p}}\left(\frac{W_{p}}{P_{a}}\right)^{1 / a}
$$

où $\mathrm{q}$, $\mathrm{a}$ et $\mathrm{b}$ sont des constantes dépendant de $\sigma_{3}$ et de $W_{\text {pmax. }}$. Elles ont pour expression :

$q=\left[\frac{\log \left(\frac{W_{p \max }}{W_{p 60}}\right)-\left(1-\frac{W_{p 60}}{W_{p \max }}\right) \log e}{\log \left(\frac{\eta_{1}}{f_{p 60}}\right)}\right]$

$a=\eta_{1}\left(e \frac{P_{a}}{W_{p \max }}\right)^{1 / q}$ et $b=\frac{1}{q W_{p \max }}$ où $\mathrm{f}_{\mathrm{p} 60}=0,6 \eta_{1} ; \mathrm{W}_{\mathrm{p} 60}=\mathrm{F}_{\mathrm{p}}\left(\mathrm{f}_{\mathrm{p} 60}\right)$.

En pratique, $q$ et $W_{\text {pmax }}$ varient en fonction de la pression de confinement $\sigma_{3}$ selon les lois suivantes:

et

$$
\mathrm{W}_{\mathrm{pmax}}=\mathrm{p} \mathrm{P}_{\mathrm{a}}\left(\frac{\sigma_{3}}{\mathrm{P}_{\mathrm{a}}}\right)^{1}
$$

$$
\mathrm{q}=\alpha+\beta \frac{\sigma_{3}}{\mathrm{P}_{\mathrm{a}}}
$$

où $\alpha$ et $\beta$ sont donc respectivement la pente et l'ordonnée à l'origine de la courbe $\mathrm{q}\left(\frac{\sigma_{3}}{\mathrm{P}_{\mathrm{a}}}\right)$.

On peut déterminer $\Delta \lambda_{\mathrm{p}}$ de la même manière que $\Delta \lambda_{c}$ à partir de l'incrément de travail plastique :

$$
d W_{p}=\left(\frac{d f_{p}}{f_{p}}\right)\left(\frac{1}{\frac{1}{q W_{p}}-b}\right)
$$

\subsection{Détermination des paramètres de la loi de LADE}

Les valeurs des paramètres, qui sont tous adimensionnels, peuvent être déterminées en utilisant les résultats d'essais de compression isotrope et de compression triaxiale.

\subsubsection{Détermination de $K$ (coefficient du module de rigidité) et $n$ (exposant)}

A partir des caractéristiques de la courbe déviateur des contraintes-déformations obtenues lors d'essais de compression triaxiale pour différentes pressions de confinement $\sigma_{3}, \mathrm{E}$ est déterminé expérimentalement à l'aide d'un chargement suivi d'un déchargement. Le tracé de la courbe $\log (E)=f\left(\log \left(\frac{\sigma_{3}}{P_{a}}\right)\right)$, comp-
te tenu de la relation:

$$
\log (E)=\log \left(K P_{a}\right)+n \log \left(\frac{\sigma_{3}}{P_{a}}\right)
$$

conduit aux deux coefficients Log $\left(\mathrm{KP}_{\mathrm{a}}\right)$ et $\mathrm{n}$ respectivement l'ordonnée à l'origine et pente de la droite de variable $\log \left(\frac{\sigma_{3}}{P_{\mathrm{a}}}\right)$.

\subsubsection{Coefficient de POISSON}

En général $\nu$ est proche de 0,2 valeur qu'on utilisera par la suite.

\subsubsection{Module de consolidation C et exposant de consolidation $p$}

Ces coefficients sont déterminés à l'aide d'essais de compression isotrope. Le travail plastique de consolidation $W_{c}$ et sa variation en fonction de $\sigma_{3}$ est déter- 
minée à partir des valeurs expérimentales de $\sigma_{3}$ et de $\epsilon_{p}$.

$$
\log \left(W_{c}\right)=\log \left(C P_{a}\right)+p \log \left(\frac{f_{c}}{P_{a}^{2}}\right)
$$

les valeurs de $\log \left(C \mathrm{P}_{\mathrm{a}}\right)$ et de $\mathrm{p}$ représentent l'ordonnée à l'origine et la pente de la droite de variable $\log \left(\frac{\mathrm{f}_{\mathrm{c}}}{\mathrm{P}_{\mathrm{a}}^{2}}\right)$.

\subsubsection{Détermination de $\eta_{1}$ et $m$}

A l'état limite de rupture $\left(\mathrm{f}_{\mathrm{p}}=\eta_{1}\right)$, la représentation de la droite:

$$
\log \left(\frac{I_{1}^{3}}{I_{3}}-27\right)=m \log \left(\frac{I_{1}}{P_{a}}\right)-\log \left(\eta_{1}\right)
$$

donne $\eta_{1}$ (paramètre de rupture) et $\mathrm{m}$ (paramètre du matériau).

\subsubsection{Détermination de $R, S$ et $T$}

Ces coefficients sont déduits de la représentation graphique de $\eta_{2}$ et de l'expression

$$
\eta_{2}=S f_{p}+R \sqrt{\frac{\sigma_{3}}{P_{a}}}+T
$$

avec

$$
f_{p}=\left(\frac{I_{1}^{3}}{I_{3}}-27\right)\left(\frac{I_{1}}{P_{a}}\right)^{m}
$$

Pour la représentation graphique, il est nécessaire de connaître $\nu_{\mathrm{p}}$, calculée par

$$
\nu_{\mathrm{p}}=-\frac{\delta \epsilon_{3}-\left(\delta \epsilon_{3}\right)_{e}-\left(\delta \epsilon_{3}\right)_{c}}{\delta \epsilon_{1}-\left(\delta \epsilon_{1}\right)_{e}-\left(\delta \epsilon_{1}\right)_{c}}
$$

Tandis que $\delta \epsilon_{3}$ et $\delta \epsilon_{1}$ sont connus à partir de l'essai de compression triaxiale; $\left(\delta \epsilon_{i j}\right)_{e}$ et $\left(\delta \epsilon_{i i}\right)_{c}$ peuvent être obtenus à partir des paramètres calculés auparavant.

On peut alors calculer $\eta_{2}$ et

- représenter sa variation en fonction de $f_{p}$, la pen-

te de ces droites représente $\mathrm{S}$;

- représenter $\eta_{2}-\mathrm{S}_{2}$ en fonction de $\sqrt{\frac{\sigma_{3}}{\mathrm{P}_{\mathrm{a}}}}$,
on obtient alors $\mathrm{R}$ et $\mathrm{T}$.

\subsubsection{Détermination de $P$ et $l$}

La détermination du travail plastique total s'effectue à partir de l'essai triaxial

$$
W_{p}=\int\left(\sigma_{i j}\right)^{t}\left(d \epsilon_{i j}\right)_{p}
$$

en considérant la représentation schématique des déformations plastiques adoptée par LADE (fig. 1).
Puis on trace la courbe expérimentale $W_{p}=F_{p}\left(f_{p}\right)$ sachant que $f_{p}$ est égal à :

$$
f_{p}=\left(\frac{I_{1}^{3}}{I_{3}}-27\right)\left(\frac{I_{1}}{P_{a}}\right)^{m}
$$

La représentation de $W_{p}$ en fonction de $f_{p}$ pour différentes valeurs de la pression de confinement $\sigma_{3}$, permet de calculer les valeurs correspondantes de $\mathrm{W}_{\text {pmax }}$ en fonction de $\sigma_{3}, \mathrm{P} . \mathrm{V}$. LADE préconise une relation du type suivant:

$$
\log \left(W_{p \max }\right)=(1-1) \log \left(\frac{p}{P_{a}}\right)+1 \log \left(\sigma_{3}\right)
$$

Il s'agit d'une relation linéaire de la variable $\log \left(\sigma_{3}\right)$ donc de pente constante égale à $1, \mathrm{p}$ étant déduit de l'ordonnée à l'origine.

\subsubsection{Détermination de $\alpha$ et $\beta$}

Toujours à l'aide des courbes $W_{p}=f\left(f_{p}\right)$ pour différentes valeurs de $\sigma_{3}$, on obtient la courbe :

$\mathrm{q}=\mathrm{q}\left(\frac{\sigma_{3}}{\mathrm{P}_{\mathrm{a}}}\right)=\alpha+\beta \frac{\sigma_{3}}{\mathrm{P}_{\mathrm{a}}}$ où $\alpha$ est l'ordonnée à l'origine et $\beta$ la pente.

\section{LA LOI DE KOLYMBAS}

Cette loi propose une nouvelle approche du comportement des milieux granulaires qui n'est pas basée sur la théorie de l'élastoplasticité. Elle repose sur une seule relation tensorielle liant le taux de contrainte aux contraintes et au taux de déformation. Cette équation tient compte des déformations non élastiques des sols (plastiques ou non réversibles) mais contrairement à la théorie de la plasticité elle ne fait pas de distinction entre les déformations élastiques et les déformations plastiques ; elle n'utilise pas non plus la notion de surface d'écoulement.

En particulier :

- selon qu'il s'agisse d'un chargement ou d'un déchargement, on utilise des relations linéaires différentes (comportement irréversible):

- la raideur instantanée dépend du niveau de contrainte :

- elle utilise un état de contrainte limite caractérisé par l'annulation de la raideur pour certaines déformations particulières ;

- elle utilise une viscosité logarithmique de type Prandtl-Eyring.

Pour des raisons d'objectivité matérielle, la loi de comportement fluide de KOLYMBAS dépendant du temps utilise la dérivée de JAUMANN objective $\tau$ du tenseur des contraintes de CAUCHY T définie par $\tau=\frac{\mathrm{dT}}{\mathrm{dt}}-\Omega \mathrm{T}+\mathrm{T} \Omega$ où le tenseur des rotations $\Omega$ constitue la partie antisymétrique de la décomposition du tenseur gradient de vitesse (grad V) en par- 
tie symétrique et antisymétrique grad $V=D+\Omega$. La loi de KOLYMBAS s'écrit :

$$
\begin{gathered}
\tau=C_{1} \frac{T D+D T}{2}+C_{2} \operatorname{Tr}(T D) I \\
+C_{3} T \sqrt{\operatorname{Tr}\left(D^{2}\right)}+C_{4} T^{2} \frac{\sqrt{\operatorname{Tr}\left(D^{2}\right)}}{\operatorname{Tr}(T)}
\end{gathered}
$$

où $C_{1}, C_{2}, C_{3}$ et $C_{4}$ sont des paramètres liés au matériau et $\mathrm{Tr}$ désigne la trace du tenseur correspondant.

Les deux premiers termes sont linéaires par rapport à $\mathrm{D}$ et décrivent un comportement hypoélastique. La dissipation interne et les déformations irréversibles sont traduites par le troisième et le quatrième terme qui sont non-linéaires.

\subsection{Restriction de son champ d'application}

Puisque la dérivée par rapport au temps n'intervient que dans $\mathrm{T}$, la loi de KOLYMBAS ne peut décrire les effets cycliques ou les effets visqueux. La loi de comportement est donc valable si linfluence des chargements cycliques est négligeable. Il en résulte que pour modéliser les effets des chargements cycliques, il devient nécessaire d'introduire un paramètre supplémentaire (éventuellement tensoriel). Cette modification n'a pas encore été introduite dans la loi. A contrario, les phénomènes visqueux peuvent d'ores et déjà être pris en compte en ajoutant le terme :

$$
\frac{A D_{2}}{\sqrt{B^{2}+\operatorname{Tr}\left(D^{2}\right)}}
$$

où $\mathrm{A}$ et $\mathrm{B}$ sont deux paramètres liés au matériau et $D_{2}=\epsilon_{i j}$.

\subsection{Détermination des paramètres de la loi}

La loi dépend de quatre paramètres, séparément sans signification particulière, mais permettant de décrire ensemble un phénomène donné. On peut les déterminer à partir d'un seul essai en compression triaxiale classique. La courbe contraintes-déformations permet de calculer la pente à l'origine $\mathrm{E}_{0}$ et l'angle de frottement interne $\Phi$; la courbe variation de volumedéformation permet de calculer l'angle de dilatance à l'état limite $\beta$. Cependant pour un milieu granulaire KOLYMBAS fixe $\beta_{0}$ égal à $-45^{\circ}$, ce qui constitue une hypothèse simplificatrice (que nous serons amené à discuter par la suite) permettant ainsi d'obtenir simplement les valeurs de certaines variables puis les paramètres du modèle à partir des courbes suivantes :

$$
\begin{aligned}
& \sin \Phi=\max \frac{\sigma_{1}-\sigma_{3}}{\sigma_{1}+\sigma_{3}} ; a=\frac{1+\sin \Phi}{1-\sin \Phi} ; \\
& b_{0}=\frac{1}{2}\left(1+\operatorname{tg} \beta_{0}\right)=0 ; \\
& b=\frac{1}{2}(1+\operatorname{tg} \beta) ; c=\sqrt{1+2 b^{2}} ; \\
& d=\frac{a-1}{a+1}[(2 b-a)(2 a+1)+3 a c]
\end{aligned}
$$

Ces différents éléments permettent d'avoir accès aux paramètres du modèle:

$$
\begin{aligned}
C_{1} & =\frac{E_{0}}{\sigma_{3}} ; \\
C_{2} & =C_{1} \frac{a}{b}\left[1+b-\frac{3}{a+2}(1+a b)\right] \\
C_{3} & =-\frac{C_{1}}{c d}\left[(2 b-a)(a+b)+\frac{3 a c}{a+2}(a b+1)\right] ; \\
C_{4} & =3\left(C_{2}-C_{3}\right)
\end{aligned}
$$

L'hypothèse de KOLYMBAS supposant que $\beta_{0}=$ $-45^{\circ}$ n'est en fait pas toujours vérifiée.

En effet puisque

$$
\begin{aligned}
& \operatorname{tg}\left(\beta_{0}\right)=\frac{\Delta \epsilon_{y}}{\Delta \epsilon_{1}}=1+2 \frac{\Delta \epsilon_{3}}{\Delta \epsilon_{1}} \text {, on aurait dans } \\
& \text { ce } \operatorname{cas} \frac{\Delta \epsilon_{3}}{\Delta \epsilon_{1}}=0 \text { en } \epsilon_{1}=0 .
\end{aligned}
$$

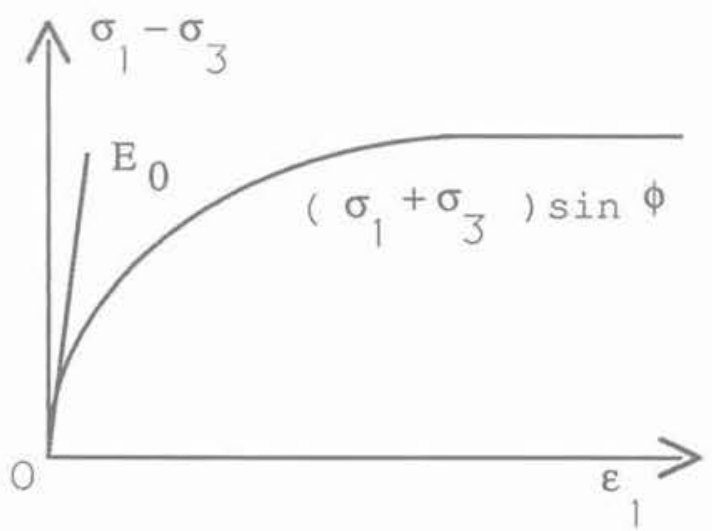

Fig. 2. - Courbe contraintes-déformations. Fig. 2. - Stress-strain curve.

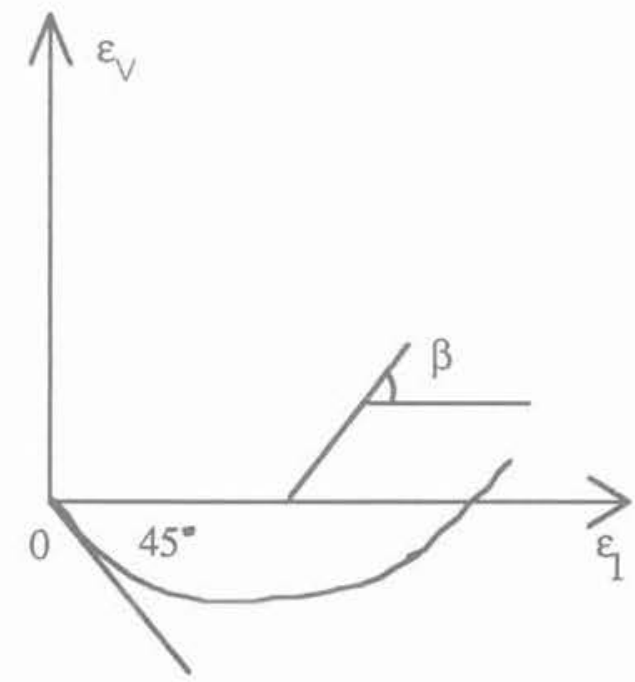

Fig. 3. - Courbe variation de volume-déformation. Fig. 3. - Volumetric-strain curve. 
Les résultats expérimentaux de C. KAMMACHER [8] obtenus sur du blé et du colza montrent clairement que la déformation latérale $\epsilon_{3}$ n'est pas négligeable devant $\epsilon_{1}$ au début de l'essai triaxial. Ce résultat a d'ailleurs été récemment observé par KOLYMBAS.

\subsubsection{Détermination expérimentale de la courbe contrainte-déformation}

L'essai triaxial classique fournit la courbe $\sigma_{1}-\sigma_{3}$ (déviateur de contrainte) en fonction de $\epsilon_{1}$. La connaissance de la contrainte de confinement permet de déterminer $\sigma_{1}$ en fonction de $\epsilon_{1}$.

Les courbes contraintes-déformations suivantes ont été obtenues par le modèle de KOLYMBAS sur divers matériaux :

Courbe contrainte-déformation

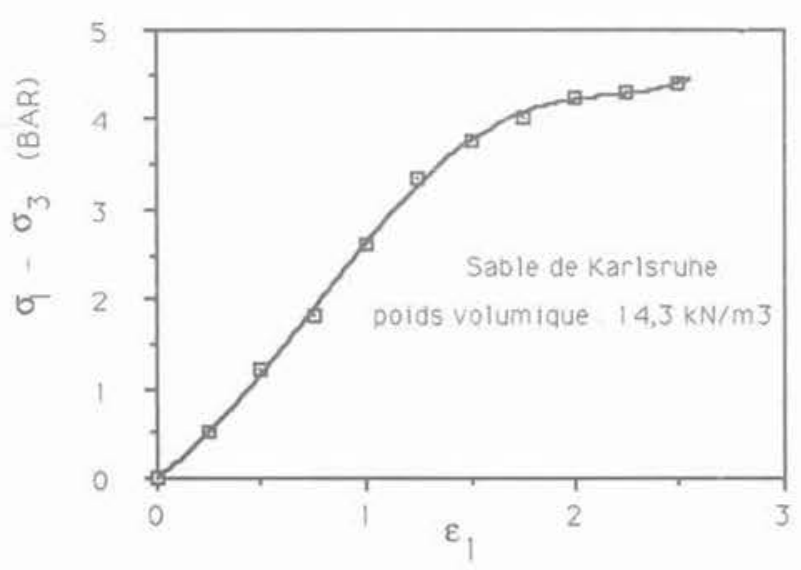

Fig. 4. - Courbe contraintes-déformations (sable de Karlsruhe). Fig. 4. - Stress-strain data (Karlsruhe sand).

Courbe contrainte-déformation

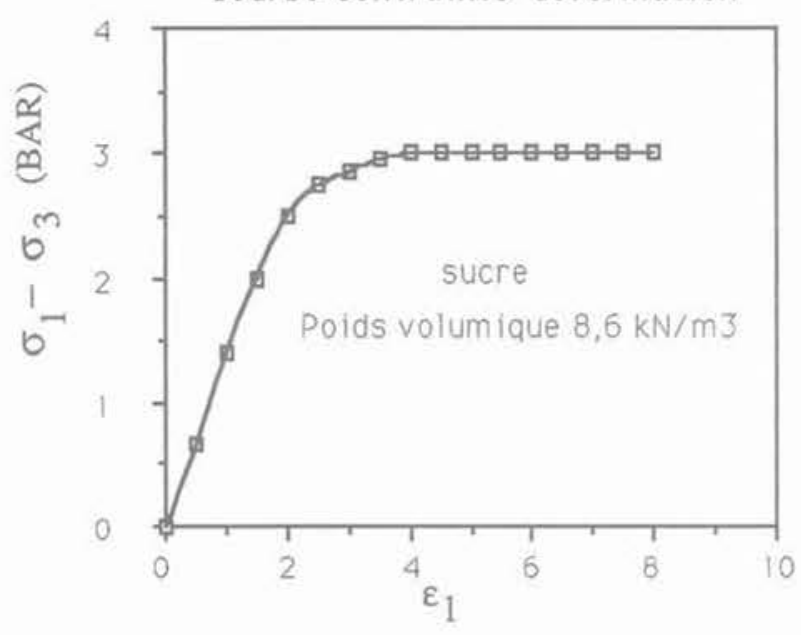

Fig. 5. - Courbe contraintes-deformations (Sucre).

Fig. 5. - Stress-strain curve (sugar).

Remarques concernant les résultats numériques obtenus :

- les courbes numériques ci-dessus présentent toutes un point d'inflexion plus ou moins marqué près de l'origine, ce qui 'est pas vérifié expérimentalement ;

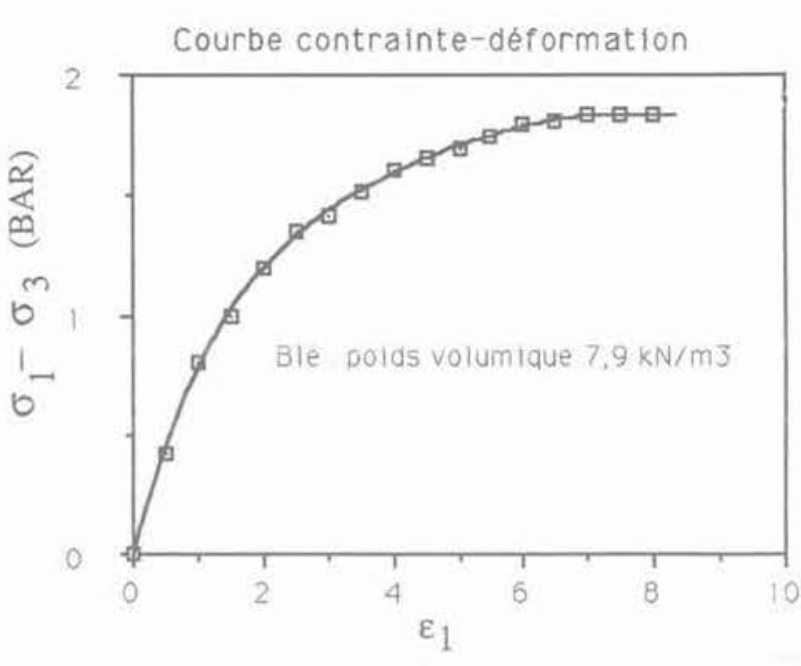

Fig. 6. - Courbe contraintes-déformations (Blé). Fig. 6. - Stress-strain curve (wheat)

- la pente à l'origine (rigidité instantanée au début de l'essai) est nettement trop faible, ce qui conduit à des difficultés pour la détermination des paramè. tres de la loi :

- la loi de KOLYMBAS, contrairement à la loi de LADE ne permet pas de décrire le comportement du matériau après la rupture ;

- pour calculer les paramètres $C_{1}, C_{2}, C_{3}$ et $C_{4}$ KOLYMBAS suppose que l'échantillon ne subit pas de déformation latérale au début de l'essai, ce qui n'est pas tout à fait exact comme nous l'avons vu plus haut.

La loi de KOLYMBAS présente d'autre part un autre inconvénient parce que pour un matériau donné les quatre paramètres $\mathrm{C}_{1}, \mathrm{C}_{2}, \mathrm{C}_{3}, \mathrm{C}_{4}$ dépendent de la densité et de la contrainte de confinement.

Ces remarques ne signifient pas pour autant que la loi de KOLYMBAS est à rejeter, bien au contraire. Elle présente, en effet, de grands avantages numériques par le petit nombre de ses paramètres et l'absence de notions de surfaces de charges difficiles à mettre en œuvre. Elle donne de bons résultats (EIBL et ROMBACH [3], [4], [5]).

\section{NATURE DES ESSAIS EXPÉRIMENTAUX}

\subsection{Matériel expérimental}

Les mesures obtenues à la base expérimentale de Chartres [1], [9] concernent deux silos de caractéristiques et de dimensions suivantes.

La cellule « $\mathrm{C}$ " à fond plat est constituée d'un silo cylindrique à parois rugueuses de diamètre $8 \mathrm{~m}$ et de hauteur $12,50 \mathrm{~m}$. La cellule « $\mathrm{D}$ * également à fond plat est constituée d'un silo cylindrique à parois lisses, réalisées par sertissage hélicoïdal d'une bande de tôle en acier galvanisé. Elle a un diamètre de $6 \mathrm{~m}$ et une hauteur de $8 \mathrm{~m}$. Chaque silo est rempli parun jet 
central situé sur son ouverture supérieure. Une trappe d'évacuation est située au centre de sa base.

\subsection{Protocole expérimental}

Les mesures à l'intérieur de la matière ensilée étant, lorsqu'elles sont possibles, très imparfaites voir impossibles sur le site de Chartres, nous avons da situer les modèles par rapport aux quantités mesurables expérimentalement: les contraintes en paroi. A partir d'un tel calage le programme peut donner les valeurs des grandeurs mécaniques à l'intérieur de la matière ensilée. La connaissance de ces contraintes permet de connaitre de manière fiable (ce qui n'est pas le cas expérimentalement) les variations de contraintes en fonction du rayon. Les contraintes exercées par la matière ensilée sur les parois sont recueillies au moyen de capteurs spéciaux [1], mesurant la pression horizontale $\sigma_{\mathrm{x}}$ (normale à la paroi verticale) et la contrainte tangentielle à la paroi donnant la contrainte de frottement $\tau$.

\section{COMPARAISON DES ESSAIS NUMÉRIQUES ET EXPÉRIMENTAUX}

\subsection{Comparaison des contraintes}

Sur la figure 7 sont représentées les variations de contraintes horizontales en fonction de la hauteur d'ensilage du silo * $\mathrm{D}$ " obtenues dans le cas du blé, d'une part à l'aide du programme SILO, d'autre part à l'aide des résultats expérimentaux.

En général il existe une bonne concordance entre les résultats expérimentaux et numériques. Cette concordance est particulièrement nette pour le calcul réalisé à l'aide de la loi de comportement de KOLYMBAS où les écarts sont très petits (environ $5 \%$ ). Elle est un peu moins évidente avec la loi de LADE où l'on observe des écarts de $15 \%$ environ.

Il est à noter cependant que le modèle numérique prédit une forte augmentation de la contrainte horizontale au fond du silo, ce qui est mis en défaut par les résultats expérimentaux qui montrent une pression beaucoup plus faible. Ces différences seraient vraisemblablement atténuées si les paramètres des lois avaient été déterminés au laboratoire à partir d'essais triaxiaux effectués dans la gamme de contraintes qui s'exercent réellement dans les silos étudiés.

\subsection{Comparaison des rapports de pressions pariétales}

Le coefficient $\lambda$ désigne le rapport de la contrainte horizontale sur la contrainte verticale. Les figures 8 et 9 montrent qu'il est approximativement constant sur toute la hauteur du silo pour chacune des deux lois de comportement considérées. Sa variation dépend peu de la matière ensilée (fig. 9).

Dans le cas de la cellule « $D$ »les valeurs de $\lambda$ calculées avec la loi de LADE dépassent en moyenne les valeurs expérimentales de $15 \%$. Par contre celles

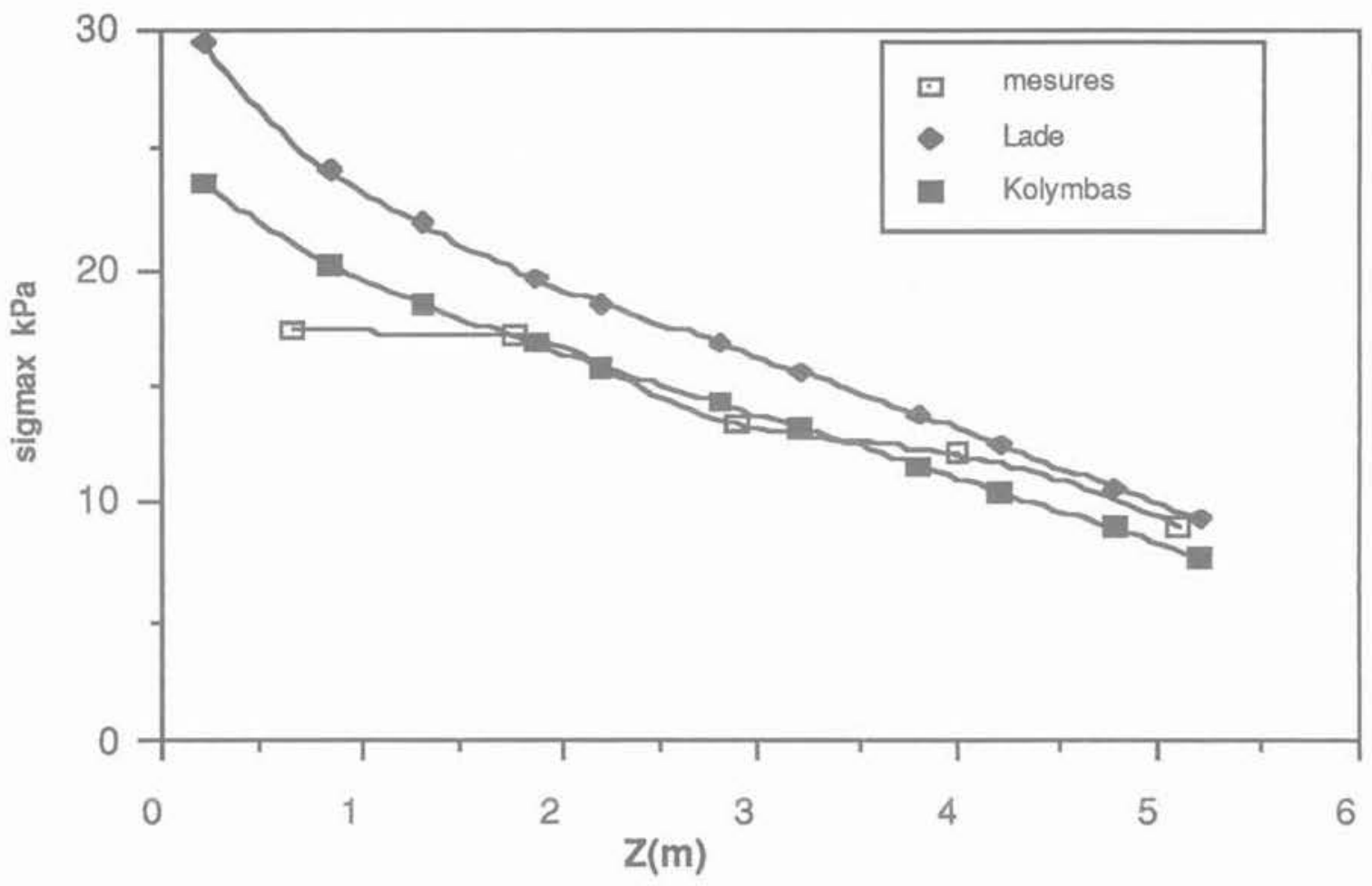

Fig. 7. - Variation de la contrainte horizontale en fonction de la profondeur (LADE, KOLYMBAS, mesures). Fig. 7. - Horizontal stress distribution on the wall (LADE, KOLYMBAS, measure). 


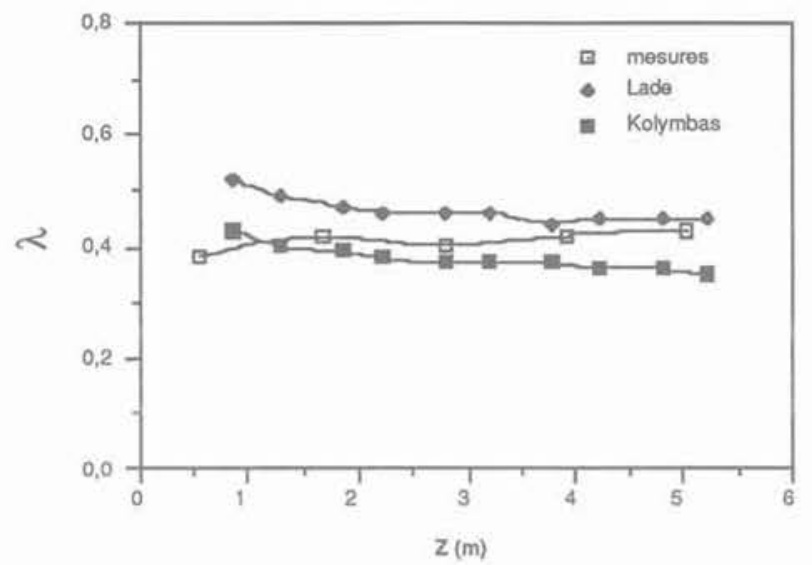

Fig. 8. - Variation de $\lambda$ en fonction de la hauteur de cellule " $D$ " matière ensilée : blé.

Fig. 8. - Variation of $\lambda$ with the height of the cell " D " ensiled material: wheat.

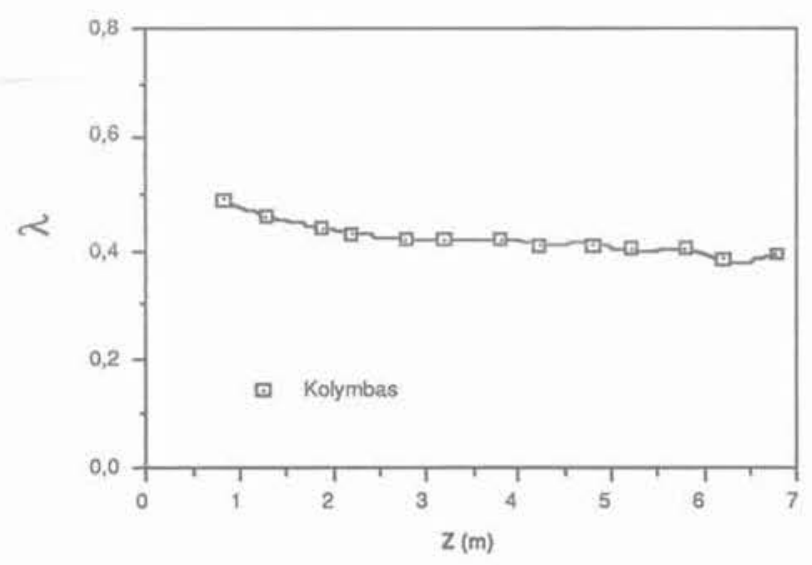

Fig. 9. - Variation de $\lambda$ en fonction de la hauteur de cellule " $D$ " matière ensilée: lupolen.

Fig. 9. - Variation of $\lambda$ with the height of the cell " $D$, ensiled material : lupolen.

obtenues avec la loi de KOLYMBAS sont inférieures aux valeurs expérimentales de $7 \%$ en moyenne.

Pour la cellule \& $C$ » nous n'avons exploité que la loi de KOLYMBAS. Les valeurs de $\lambda$ obtenues dépassent légèrement les résultats expérimentaux (fig. 10). On peut donc conclure que la loi de LADE conduit à des valeurs de contraintes horizontales un peu trop fortes et à des valeurs de contraintes verticales un peu faibles, alors que la loi de KOLYMBAS prédit le contraire. Cependant les écarts ne sont pas très importants.

\section{ESSAIS NUMÉRIQUES : INFLUENCE DES PARAMĖTRES GÉOMÉTRIQUES ET MÉCANIQUES SUR LES CONTRAINTES}

L'étude expérimentale sur le site de Chartres a permis la mise en évidence partielle de l'influence des

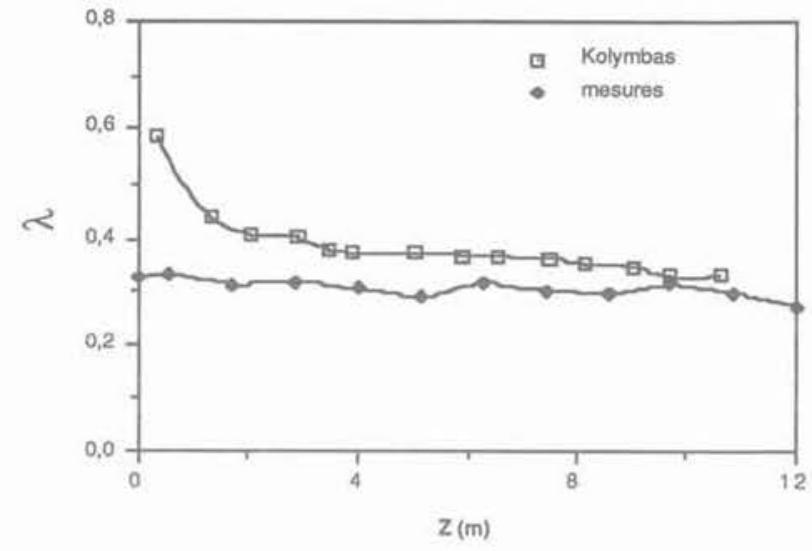

Fig. 10. - Variation de $\lambda$ en fonction de la hauteur de la cellule " $C$ " matière ensilée : blé.

Fig. 10. - Variation of $\lambda$ with the height of the cell " $C$. ensiled material : wheat.

paramètres géométriques (élancement) et mécaniques (coefficient de frottement en paroi et rigidité de la paroi) sur les contraintes. Nous avons cependant effectué un certain nombre d'essais numériques en faisant varier, pour une cellule donnée, d'une part le coefficient de frottement, et d'autre part la rigidité des parois.

\subsection{Influence du frottement en paroi (loi de LADE)}

La modélisation du frottement en paroi s'effectue à laide de la loi classique de frottement de COULOMB, reposant sur un coefficient de frottement constant ; reliant la contrainte normale horizontale $\sigma_{\mathrm{x}}$ et verticale tangentielle $\tau$ par:

$$
\tau=\sigma_{\mathrm{x}} \operatorname{tg} \Phi
$$

où $\Phi$ désigne l'angle de frottement en paroi et $\mu=\operatorname{tg} \Phi$ le coefficient de frottement.

Cette loi de frottement est utilisée dans le programme d'éléments finis comme condition limite le long des parois verticales. Le rapport $\tau / \sigma_{x}$ est comparé à chaque calcul avec $\mu$ puis corrigé également si nécessaire.

Les essais numériques pour différentes valeurs du coefficient de frottement en paroi en utilisant la loi de $\mathrm{LADE}$, ont mis en évidence l'influence du frottement en paroi sur les contraintes, d'une part à l'intérieur de la matière ensilée, d'autre part en paroi.

\subsubsection{Influence du frottement en paroi sur les contraintes à l'intérieur de la matière ensilée}

Dans le cas d'une paroi parfaitement lisse $(\mu=0)$, la distribution des contraintes est identique à celle d'un fluide au repos. Les contraintes principales majeures et mineures sont respectivement verticales et horizontales (fig. 11). Lorsque le coefficient de frottement en paroi augmente (fig. 12, 13, 14) ; les contraintes prin- 


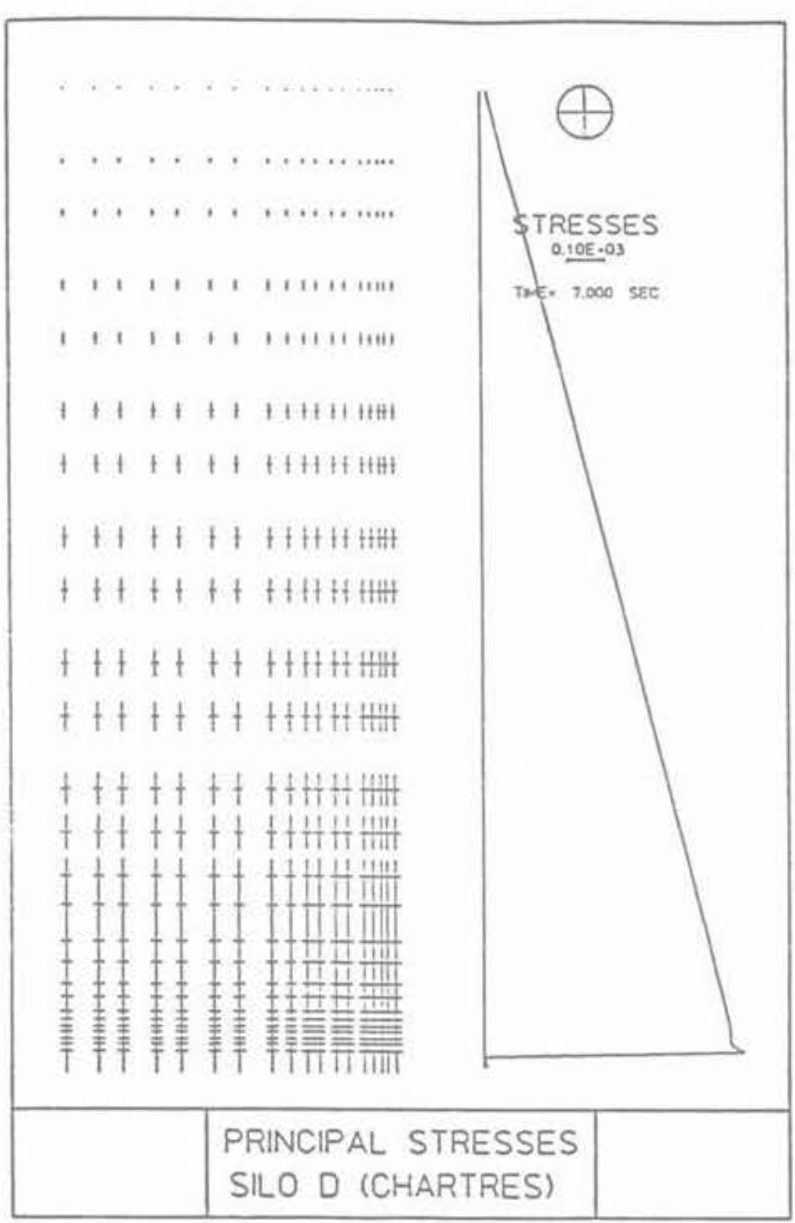

Fig. 11. - Contraintes principales: $\mu=0$. Fig. 11. - Principal stresses: $\mu=0$.

cipales varient en fonction de la distance à l'axe de symétrie vertical du silo.

\subsubsection{Influence du frottement en paroi} sur les contraintes verticales au fond du silo.

Lorsque la paroi est parfaitement lisse $(\mu=0)$ l'ensemble du poids de la matière ensilée est portée par le fond du silo et comme pour un fluide au repos, la pression verticale ne dépend pas de la distance à l'axe de symétrie vertical du silo (voir tableau 1). La contrainte verticale uniforme au fond du silo est donnée dans ce cas par $\sigma_{z}=\gamma \mathrm{h}$, où $\gamma$ désigne le poids volumique de la matière.

Tableau 1. - Influence de $\mu$ sur la contrainte verticale $\sigma_{\mathrm{z}}$ au fond $d u$ silo.

Table 1. - Influence of $\mu$ on the vertical stress $\sigma_{2}$.

\begin{tabular}{|c|r|r|r|r|r|r|}
\hline$\mu$ & 0 & 0,1 & 0,2 & 0,3 & 0,4 & 0,5 \\
\hline$\sigma_{z} \%$ & 100 & 90,2 & 79,2 & 70,6 & 65,6 & 58,9 \\
\hline Pf $\%$ & 0 & 9,8 & 20,8 & 29,4 & 34,3 & 41,1 \\
\hline
\end{tabular}

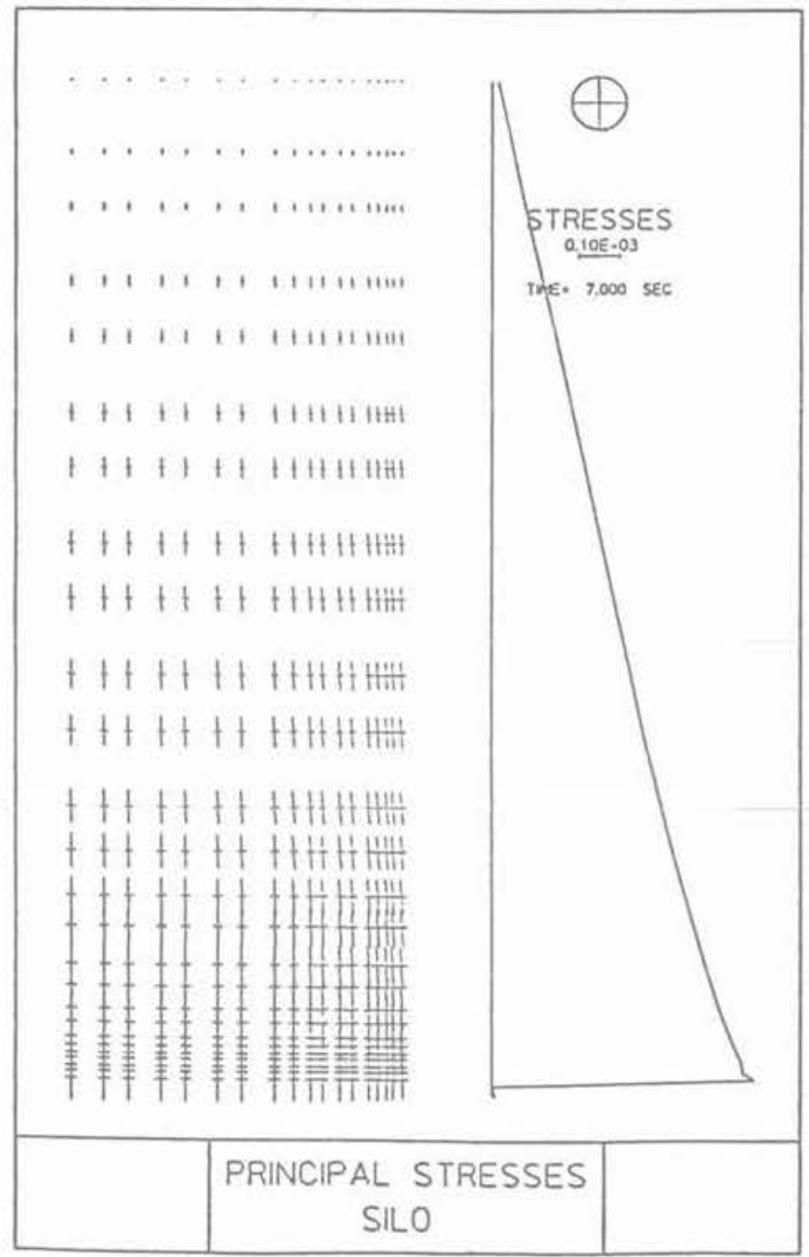

Fig. 12. - Contraintes principales: $\mu=0,2$. Fig. 12. - Principal stresses: $\mu=0.2$

Lorsque $\mu$ augmente la pression verticale sur le fond diminue, une partie du poids de la matière ensilée $p_{f}$ est alors supportée par les parois du silo.

Les figures 15 et 16 montrent une très forte influence du frottement en paroi sur la distribution de la contrainte verticale.

\subsubsection{Influence du frottement en paroi sur les contraintes horizontales}

Les figures 17 et 18 présentent l'influence du frottement en paroi sur les contraintes horizontales. Dans le cas d'une paroi lisse, on observe une évolution linéaire des contraintes en fonction de $\mathrm{z}$. Lorsque $\mu$ augmente la courbe profondeur-contrainte calculée s'approche de plus en plus de la forme exponentielle donnée par la théorie classique de JANSSEN. Toutefois une augmentation très rapide de la contrainte horizontale se manifeste lorsqu'on s'approche du fond quelque soit la géométrie du silo. Ce phénomène n'a pas encore pu être vérifié expérimentalement. 


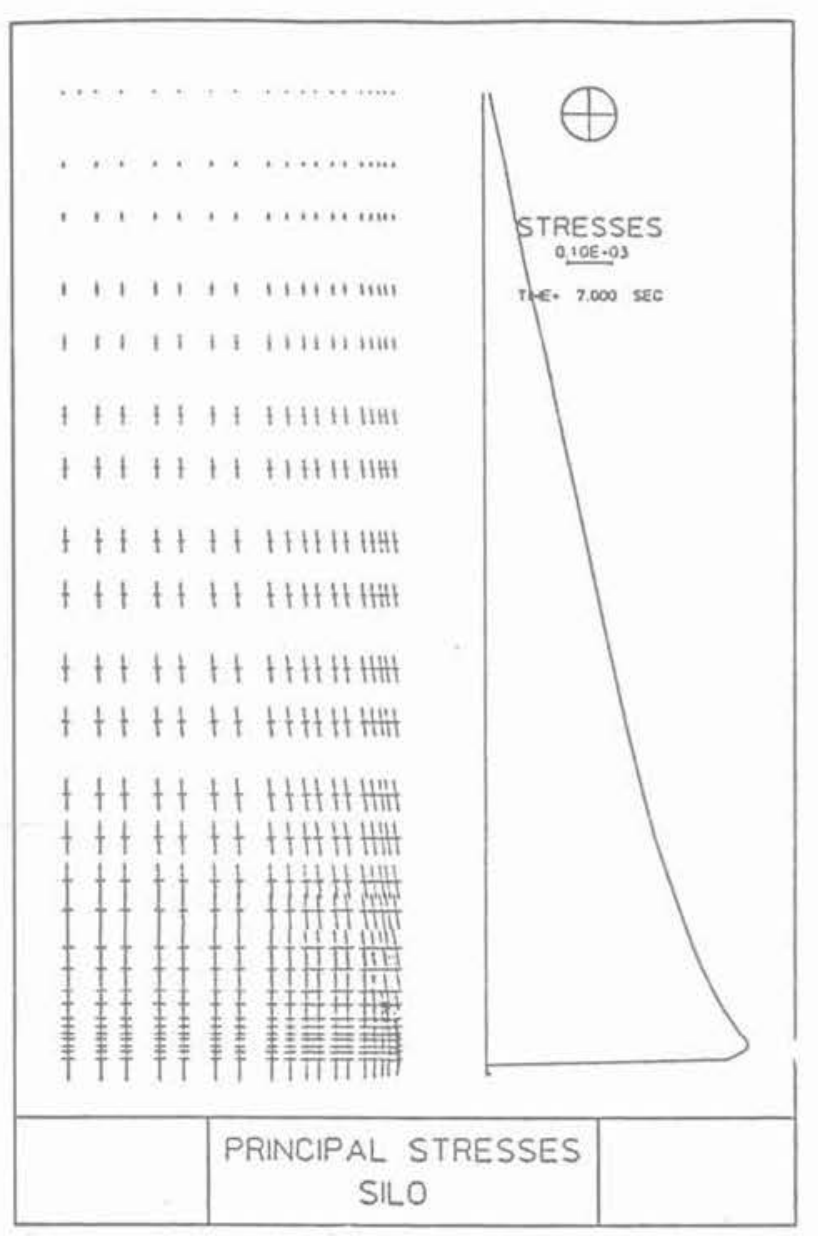

Fig. 13. - Contraintes principales : $\mu=0,4$. Fig. 13. - Principal stresses: $\mu=0.4$.

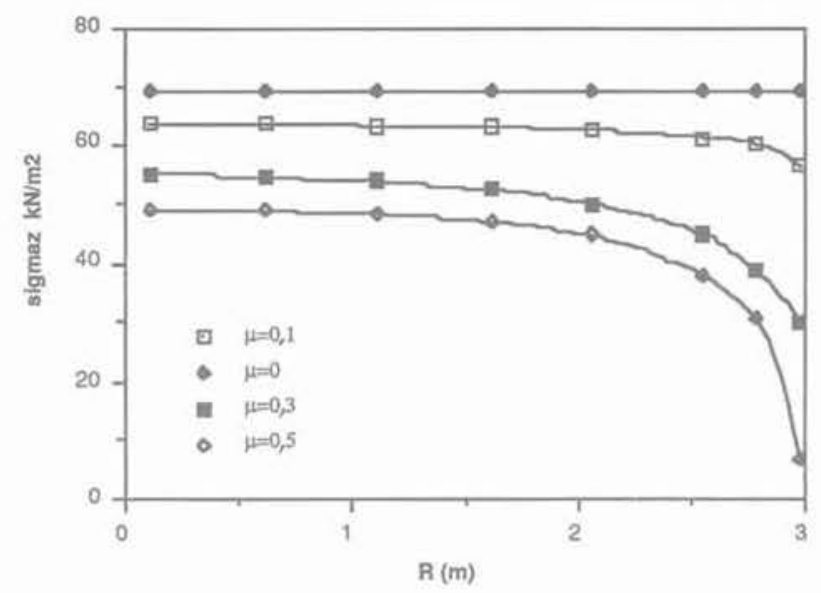

Fig. 15. - Distribution de la contrainte verticale sur le fond: blé.

Fig. 15. - Vertical stress distribution on the base: wheat.

\subsection{Influence de la rigidité des parois} sur les contraintes (loi de LADE)

Le programme SILO utilisé dans la modélisation de la cellule " D ", testée avec différentes rigidités (c'està-dire pour différents modules d'YOUNG), a fourni

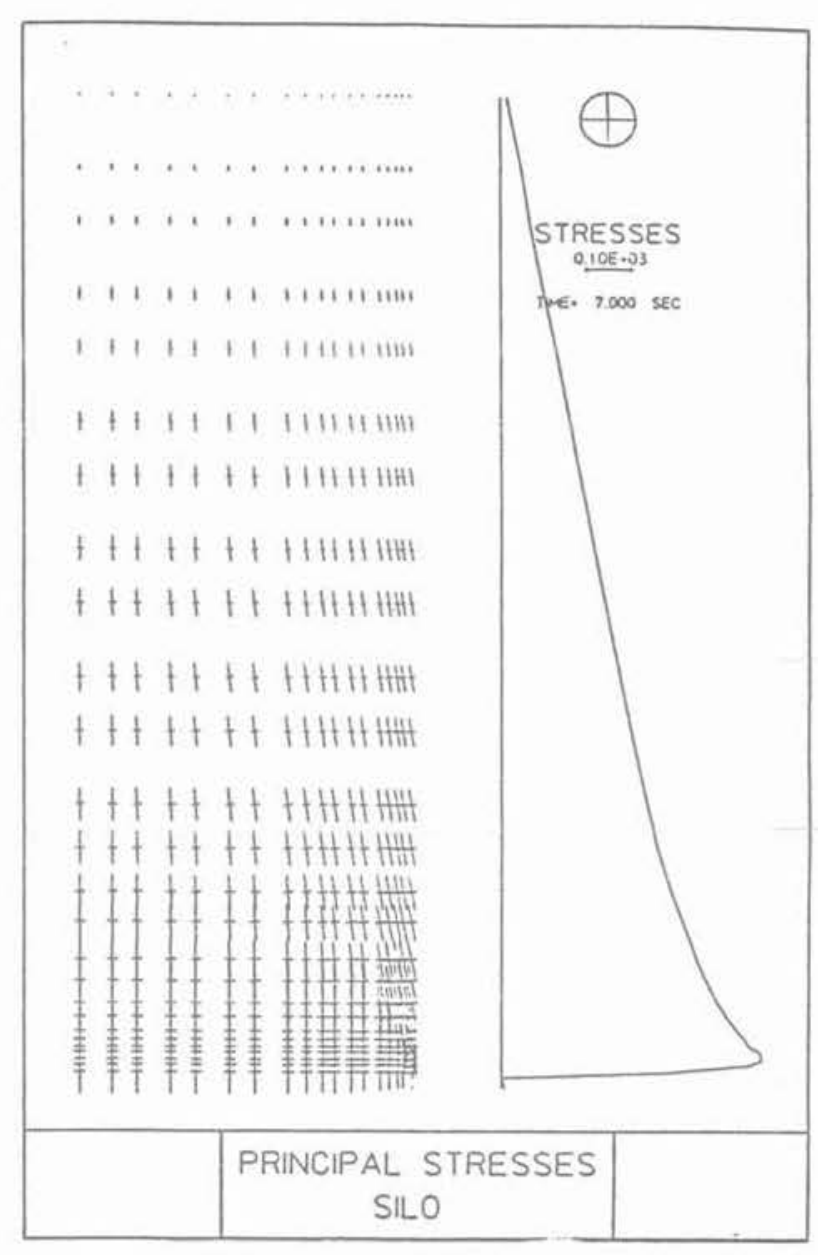

Fig. 14. - Contraintes principales: $\mu=0,5$.

Fig. 14. - Principal stresses : $\mu=0.5$.

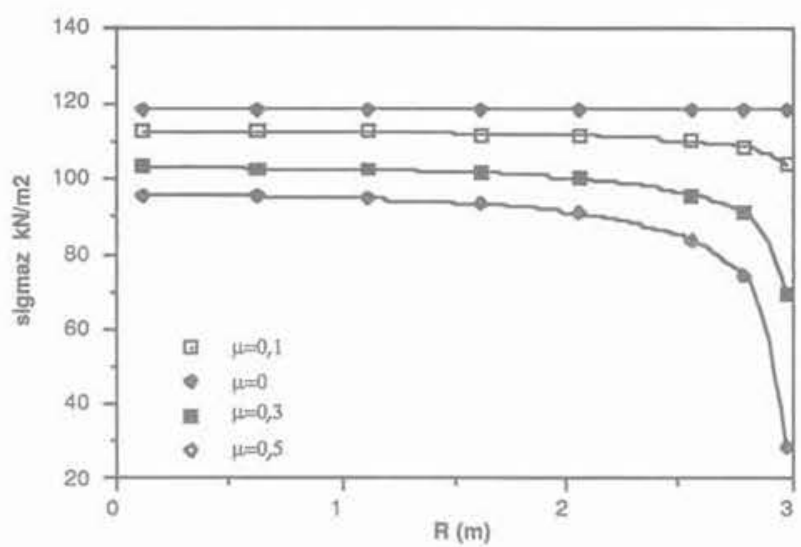

Fig. 16. - Distribution de la contrainte verticale sur le fond: sable.

Fig. 16: - Vertical stress distribution on the base: sand.

les contraintes horizontales et verticales à l'intérieur de la matière ensilée et au niveau de la paroi. Les résultats obtenus consignés dans les tableaux 2 et 3 montrent que la rigidité des parois n'a quasiment pas d'influence sur les contraintes en parois verticales et sur le fond horizontal du silo. 
Tableau 2. - Contraintes verticales sur le fond de la cellule pour différentes rigidités.

Table 2. - Vertical stress on the base of the cell for various stiffness.

\begin{tabular}{|c|c|c|c|}
\hline & \multicolumn{3}{|c|}{ Contraintes verticales } \\
\hline Rayon en $\mathrm{m}$ & $\mathrm{E}=10^{13} \mathrm{~Pa}$ & $\mathrm{E}=2,110^{11} \mathrm{~Pa}$ & $\mathrm{E}=10^{10} \mathrm{~Pa}$ \\
\hline 2,97 & 69,5 & 69,5 & 69,7 \\
2,78 & 91,1 & 91,1 & 91,1 \\
2,55 & 96,0 & 96,0 & 96,0 \\
2,05 & 100,1 & 100,1 & 100,1 \\
1,61 & 101,6 & 101,6 & 102,4 \\
1,01 & 102,5 & 102,5 & 102,8 \\
0,61 & 102,9 & 102,9 & 103,0 \\
0,11 & 103,1 & 103,1 & \\
\hline
\end{tabular}

Tableau 3. - Contraintes horizontales-profondeur pour différentes rigidités.

Table 3. - Horizontal stress-depth for various stiffness.

\begin{tabular}{|c|c|c|c|}
\hline & \multicolumn{3}{|c|}{ Contraintes horizontales } \\
\hline$Z(\mathrm{~m})$ & $\mathrm{E}=10^{13} \mathrm{~Pa}$ & $\mathrm{E}=2,110^{11} \mathrm{~Pa}$ & $\mathrm{E}=10^{10} \mathrm{~Pa}$ \\
\hline 0,84 & 27,8 & 27,8 & 27,7 \\
1,87 & 22,3 & 22,3 & 22,2 \\
2,79 & 18,5 & 18,5 & 14,9 \\
3,79 & 14,9 & 14,9 & 11,3 \\
4,79 & 11,3 & 11,3 & 7,8 \\
5,79 & 7,8 & 7,8 & 4,1 \\
6,79 & 4,1 & 4,1 & 0,8 \\
7,79 & 0,8 & 0,8 & \\
\hline
\end{tabular}

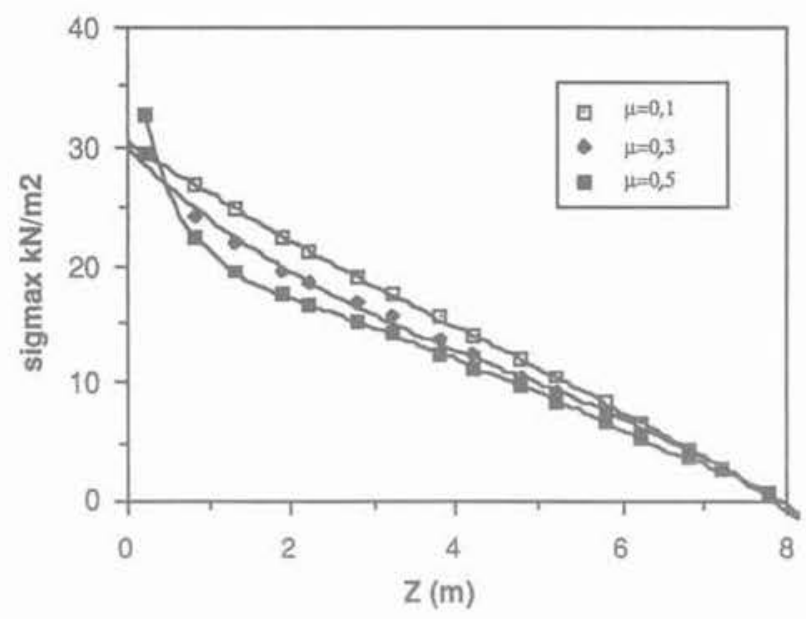

Fig. 17. - Contrainte horizontale pour différents coefficients de frottement en paroi : blé.

Fig. 17. - Horizontal stress for various wall friction coefficient : wheat.

\subsection{Etat de contraintes en paroi pour différentes matières ensilées (Loi de KOLYMBAS)}

La figure 19 présente les courbes : contrainte horizontale en paroi en fonction de la profondeur et la figure 20 les courbes : contraintes verticales en fonc-

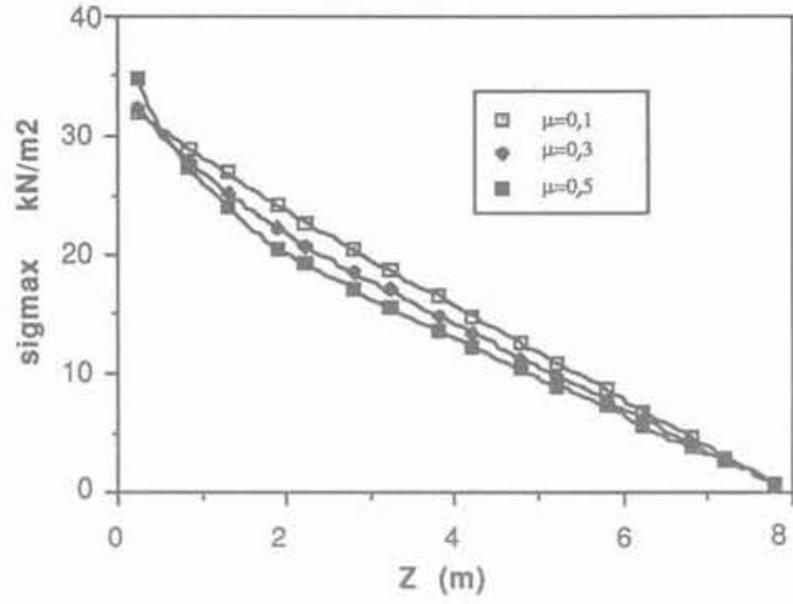

Fig. 18. - Contrainte horizontale pour différents coefficients de frottement en paroi : paroi.

Fig. 38. - Horizontal stress for various wall friction coefficient. sand:

tion du rayon au fond du silo. Elles présentent une même allure, chacune d'entre elle se déduisant de l'autre par une affinité. Les courbes correspondant aux matériaux les plus denses présentent le plus haut niveau de contraintes dans la matière ensilée aussi bien que sur les bords. On observe que la distribution de la contrainte verticale au fond du silo en fonction du rayon ne dépend pas du matériau testé. 


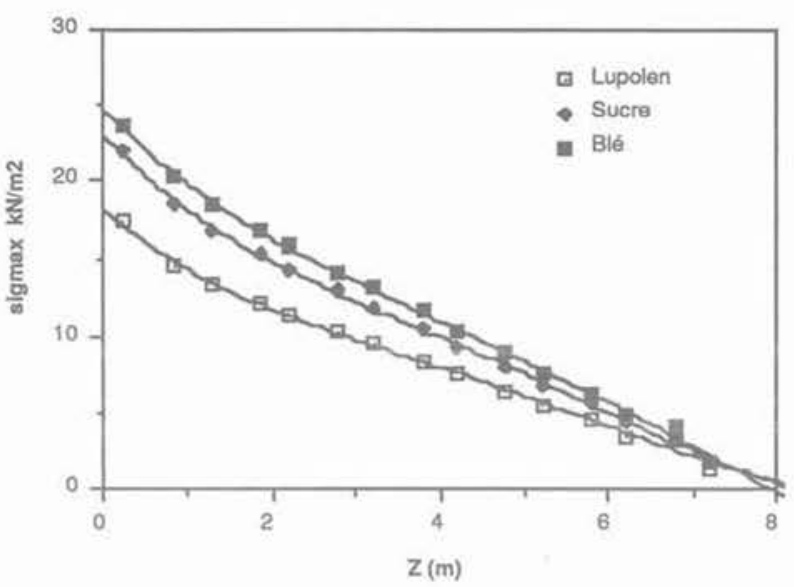

Fig. 19. - Contrainte horizontale - profondeur pour différentes matières ensilées.

Fig. 19. - Horizontal stress - depth for various ensiled materials.

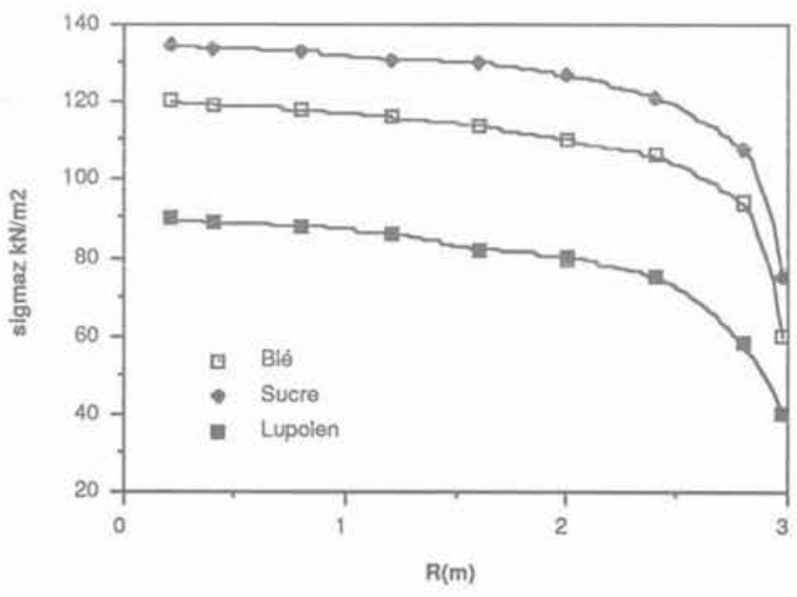

Fig. 20. - Distribution de la contrainte verticale au fond pour différentes matières ensilées.

Fig, 20. - Vertical stress distribution on the base for various ensiled materials.

\subsection{Etude de l'influence de l'élancement du silo sur les contraintes en paroi} (loi de LADE)

Les dimensions des silos influencent fortement les valeurs des contraintes exercées par la matière ensilée sur les parois du silo. Des phénomènes mécaniques de frottement en paroi, propres aux silos très élancés, pouvant conduire à l'annulation des contraintes verticales ont pu être observés. Cette remarque nous a conduit à entreprendre une étude numérique montrant l'influence des dimensions des silos et du frottement en paroi sur les contraintes dans la matière ensilée.

\subsubsection{Influence de l'élancement du silo sur les contraintes}

Les silos étudiés ont une hauteur de $12 \mathrm{~m}$ et des diamètres de $14 \mathrm{~m}, 12 \mathrm{~m}, 10 \mathrm{~m}$ et $6 \mathrm{~m}$. La figure 21 représente les variations de contraintes horizontales en fonction de la hauteur pour différents élancements de silos. On remarque que plus le silo est large par rapport à la hauteur plus l'évolution de la contrainte hori-

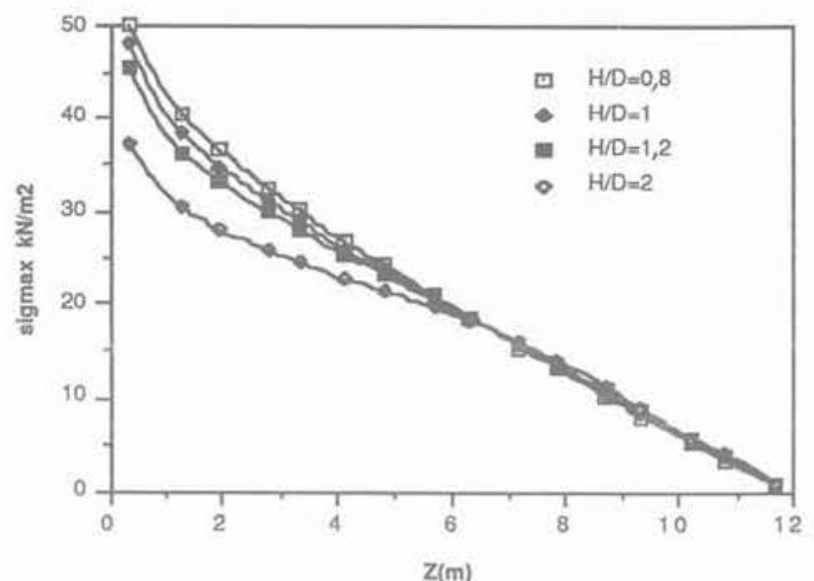

Fig. 21. - Contrainte horizontale-profondeur pour différents élancements de silos.

Fig. 21. - Horizontal stress-depth for various slenderness.

zontale en fonction de $z$ a tendance à être linéaire jusqu'au fond du silo. Dans le cas du silo de faible élancement le problème du calcul des contraintes est ramené à la théorie classique de mécanique des sols et la théorie de RANKINE s'applique bien. Il faut signaler que pour la partie supérieure du silo, le diamètre n'a aucune influence sur les contraintes horizontales.

\subsubsection{Influence du frottement en paroi sur les contraintes pour un silo très élancé}

Cette étude a été réalisée avec du sucre et du blé pour un silo de diamètre $6 \mathrm{~m}$ et une hauteur de $30 \mathrm{~m}$.

Dans le cas de la cellule très élancée, l'influence du frottement en paroi est nettement plus importante que dans un silo relativement large (ou réservoir). Ceci résulte du fait que pour un silo de très grande hauteur une plus grande partie du poids du matériau est reprise par les parois et donc non transmise au fond. Les figures 22 et 23 montrent que pour $\mu=0,5$, les contraintes horizontales sont inférieures de $30 \%$ à celles obtenues avec $\mu=0,3$.

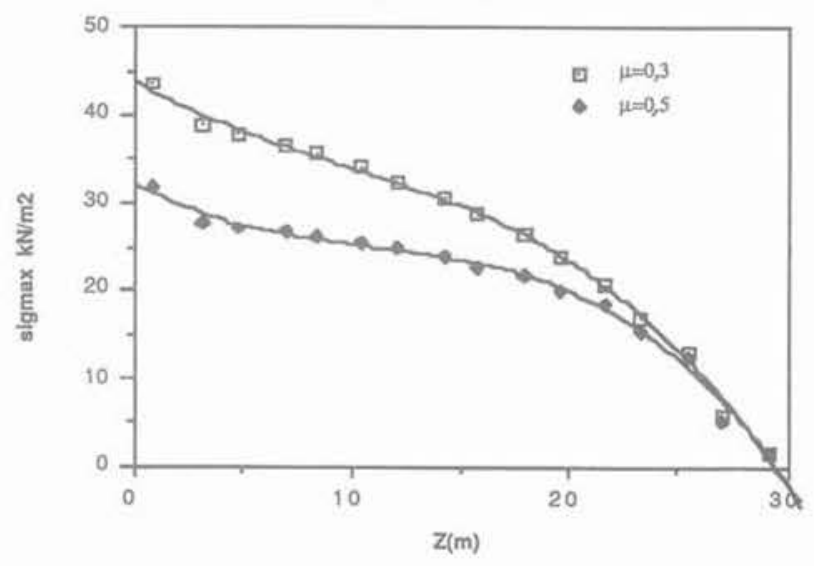

Fig. 22. - Contrainte horizontale-profondeur pour différents coefficients de frottement en paroi matière ensilée : blé.

Fig. 22. - Horizontal stress-depth for various coefficient of wall friction : ensiled material : wheat. 


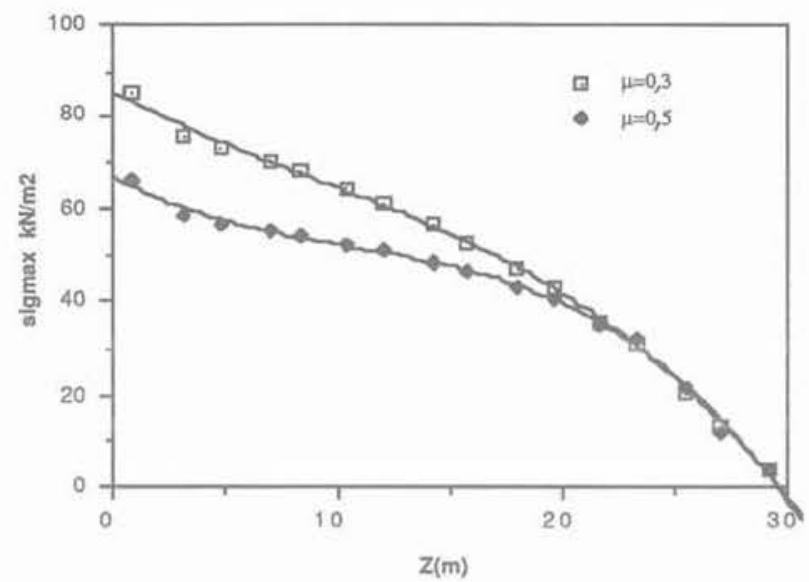

Fig. 23. - Contrainte horizontale-profondeur pour différents coefficients de frottement en paroi matière ensilée : sable.

Fig. 23. - Horizontal stress-depth for various coefficient of wall friction ; ensiled material: sand.

\subsection{Etude de l'influence du poids volumique de la matière}

Le poids de la matière agit différemment dans les silos élancés et dans les silos peu élancés. On étudie l'influence du poids de la matière en considérant l'évolution des contraintes horizontales et verticales dans le cas d'un silo de $30 \mathrm{~m}$ de hauteur et de $6 \mathrm{~m}$ de diamètre pour quatre matières ensilées:

- sable dense de Karlsruhe (SDK)

$\left(\gamma=17 \mathrm{kN} / \mathrm{m}^{3}\right) \mu=0,3$;

- sable lâche de Karlsruhe (SLK)

$\left(\gamma=14,5 \mathrm{kN} / \mathrm{m}^{3}\right) \mu=0,3$;

- sable dense de Braunschweig (SDB)

$\left(\gamma=17 \mathrm{kN} / \mathrm{m}^{3}\right) \mu=0,5$;

* sable lâche de Braunschweig (SLB)

$\left(\gamma=14,3 \mathrm{kN} / \mathrm{m}^{3}\right) \mu=0,5$.

La variation de contrainte horizontale entre le SDK $\left(\gamma=17 \mathrm{kN} / \mathrm{m}^{3}\right)$ et le SLB $\left(\gamma=14,3 \mathrm{kN} / \mathrm{m}^{3}\right)$ correspond plus ou moins à la variation de densité. On remarque ainsi sur les figures 24 et 25 que la contrainte horizontale dépend de la densité. Par contre létude des contraintes verticales au fond du silo pour les différentes matières ensilées (fig. 26) montre que la densité n'est pas le seul facteur caractéristique du matériau influençant les contraintes. En effet le SDB et le SDK ont une même densité tout en présentant des contraintes verticales différentes de $7 \%$ environ. Ceci est également vrai pour un coefficient de frottement en paroi $\mu=0,5$ et pour $\mu=0,3$.

\section{PERSPECTIVES ET CONCLUSION}

L'analyse comparative des résultats expérimentaux de la base de Chartres et des résultats numériques obtenus par l'exploitation des lois de comportement de LADE et de KOLYMBAS démontre lors du remplis-

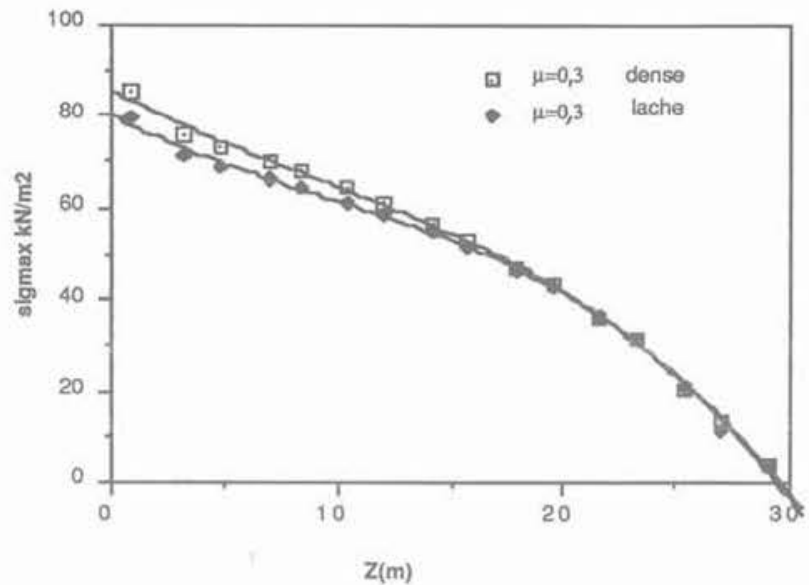

Fig. 24. - Contrainte horizontale-profondeur: sable dense et sable lâche: $\mu=0,3$. Fig. 24. - Horizontal stress-depth dense and loose sand: $\mu=0.3$.

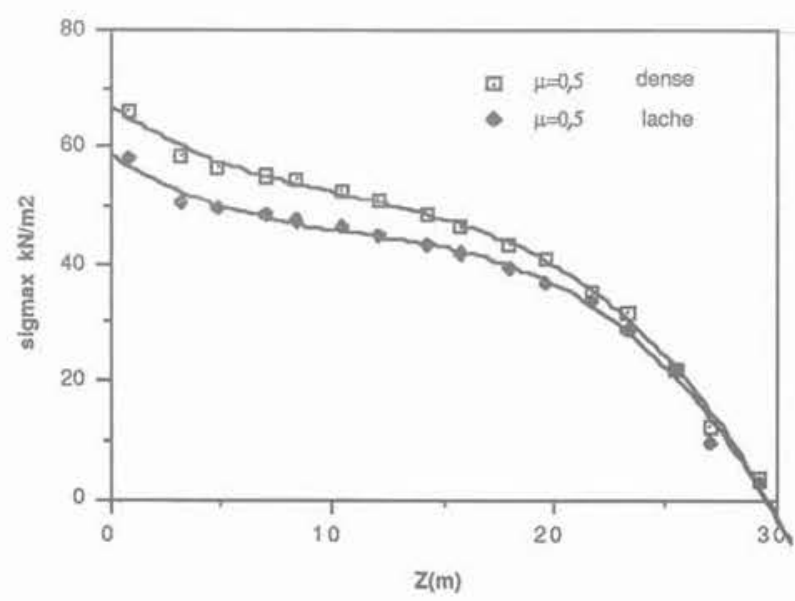

Fig. 25. - Contrainte horizontale-profondeur: sable dense et sable lâche : $\mu=0,5$. Fig. 25. - Horizontal stress-depth dense and loose sand: $\mu=0.5$.

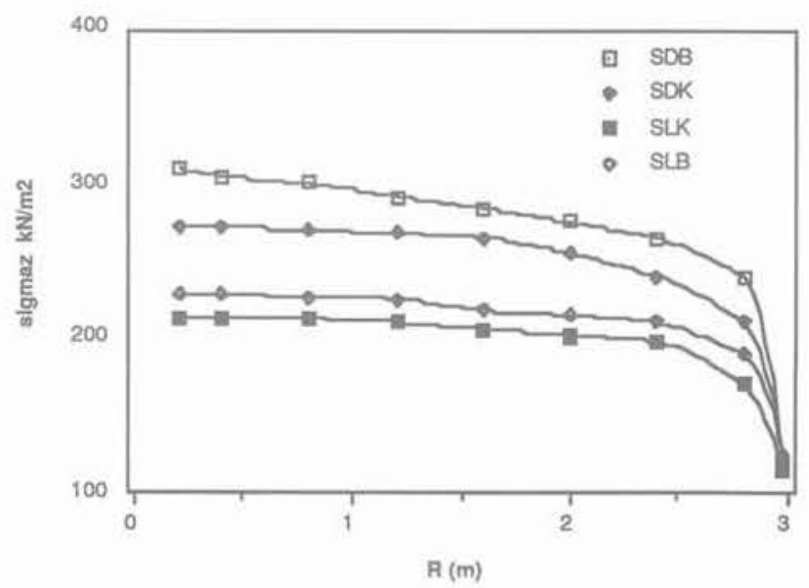

Fig. 26. - Distribution de la contrainte verticale sur le fond pour différents sables.

Fig. 26. - Vertical stress distribution on the base for various sands. 
sage des silos, une bonne approche du comportement de la matière ensilée par ces lois. Les tests numériques effectués sur différents silos mettent en évidence l'influence des paramètres géométriques (élancement) et mécaniques (coefficient de frottement en paroi et rigidité) sur l'état de contraintes. Cette simulation montre en particulier le rôle important de l'élancement et du coefficient de frottement $\mu$ dans la définition des actions de la matière sur les parois de silos.

Les résultats numériques dépendent fortement des paramètres rhéologiques utilisés dans les deux lois exploitées, il est cependant raisonnable de penser améliorer la prédiction numérique du champ de contraintes en effectuant des tests sur les matériaux en laboratoire dans des conditions de contraintes aussi proches que possible de celles rencontrées dans les silos en vraie grandeur.

\section{REMERCIEMENTS}

Nous tenons à remercier les professeurs EIBL et KOLYMBAS de l'Université de Karlsruhe pour nous avoir permis de bénéficier de l'environnement scientifique de leurs laboratoires.

\section{BIBLIOGRAPHIE}

[1] BROZZETTI . (1989), Description de la base expérimentale d'essais de cellules métalliques de stockage. CTICM n² 2, 1989.

[2] CAMBOU B. (1987), Analyse du domaine de validité de quelques modèles de comportement adaptés aux sols. Rapport Scientifique du GRECO rhéologie des matériaux sols, béton, roches, 1987, pp. 235-247.

[3] EIBL J., ROMBACH G. (1987), Arbeits und Ergebnisberichte fûr die Jahre 1985-1986, SFB 219, Teilprojet A1. Karlsruhe, März 1987.
[4] EIBL J., ROMBACH G. (1987), Numerical computation of velocity fields in silos, theory and application. Proceedings Silosy, Sklarska, Poreba, October 1987.

[5] EIBL J., WEIDNER L. (1987), Arbeits- und Ergebnisberichte für die Jahre 1985-1986, SFB 219, Teilprojekt A2. Karlsruhe, März 1987.

[6] GLADEN W. (1985), Numerische Untersuchung der Lasten in Silozellen beim exentrischen Ent. leeren. Dissertation (1985) Universität Karlsruhe.

(7) HÄUSSLER U. (1984), Geschwindigkeits- und Spannungsfelder beim Entleeren von Silozelle. Dissertation (1984) Universität Karlsruhe.

[8] KAMMACHER CH. (1988), Etude de la loi de comportement de $L A D E$. Identification des paramètres rhéologiques. DEA Mécanique juillet 1988.

[9] KHELIL A. (1989), Etude du champ de contraintes et de vitesses dans les silos métalliques. Thèse de Doctorat INPL, Nancy 1989.

[10] KOLYMBAS D. (1987), A constituive law of the rate type for soils and other granular materials. Proceedings Numerical methods in geomechanics, Kosice, may 1987.

[11] KOLYMBAS D., GUDEHUS G., TEJCHMAN J. (1986), Behaviour of granular materials in cylindrical silos. Powder Technology, vol. 48 , 1986.

[12] LADE P.V. (1977), Elasto-plastic stress-strain theory for cohesionless soil with curved yield surfaces. J. Solids Structures, 1977, vol. 13, 1019-1035.

[13] REMESCH J.C. (1989), Etude numérique du champ de contraintes dans les silos cylindriques lors du remplissage. DEA INPL, Nancy 1989.

[14] ROMBACH G., EIBL J. (1987), Stress and velocity fields at discharging of silos. Proceeding of Numeta, Swansea, July 1987. 